\title{
Reprocessing Models and the ASCA Spectrum of Mkn 290
}

\author{
T. J. Turner, ${ }^{1}$ I. M. George, ${ }^{1}$ T. Kallman, and T. Yaqoob ${ }^{1}$ \\ Laboratory for High Energy Astrophysics, Code 660, NASA/Goddard \\ Space Flight Center, Greenbelt, MD 20771, USA \\ P.T. Życki \\ Nicolaus Copernicus Astronomical Center, Bartycka 18, 00-716, \\ Warsaw, Poland
}

\begin{abstract}
We describe a warm absorber/emitter model that is used to model the complex X-ray spectrum of Mkn 290. The best fit to the data also includes a contribution from an ionized reflector.
\end{abstract}

A $40 \mathrm{ks} A S C A$ observation of the Seyfert 1 galaxy Mkn 290 reveals that this source has a complex spectral form, with emission and absorption features due to oxygen and iron superimposed on an underlying power-law continuum with photon index $\sim 1.9$. Mkn 290 is also found to exhibit significant flux-correlated spectral variability, predominantly below $2 \mathrm{keV}$, on a timescale of $\sim 5 \times 10^{4} \mathrm{~s}$.

The relatively strong spectral features observed in this source make it an interesting case to use for an investigation of models for reprocessing of the $\mathrm{X}$ ray continuum by ionized material. We consider ionized reprocessors based upon the XSTAR photoionization code, for geometries in and out of the line of sight. Models in either geometry provide a vast improvement over a simple absorbed power-law model.

The warm absorber/emitter model provides a good explanation of the overall spectral shape, with a column density of $N_{\mathrm{H}, z}^{*} \approx 8 \times 10^{21} \mathrm{~cm}^{-2}$ of ionized material (ionization parameter $\xi=24$ ) most likely within $\sim 10^{17} \mathrm{~cm}$ of the central source. While the data do not allow us to unambiguously determine the origin of the spectral variability, it is consistent with a drop of $\sim 25 \%$ in flux accompanied by a proportional drop in the ionization state of the warm absorber. The intense $\mathrm{K} \alpha$ line of equivalent width $\sim 500 \mathrm{eV}$ present in the source can be modeled as a broad Gaussian of FWHM $\sim 0.5 \mathrm{keV}$ or by a line profile expected from the inner regions of a relativistic accretion disk inclined at $\sim 30^{\circ}$. In both cases, the rest-frame line energy is consistent with weakly ionized iron, and cannot be explained by the warm emitter.

An ionized reflector can also model the overall spectral shape, yielding $\xi_{\text {ref }} \approx$ 80 , although it has some difficult simultaneously fitting both the iron $\mathrm{K} \alpha$ line and the soft X-ray spectral features. This model offers no simple explanation for the observed spectral variability, and the intensity of the reflected component is

\footnotetext{
${ }^{1}$ Also Universities Space Research Association.
} 
greater than that expected from a semi-infinite slab illuminated by an isotropic source.

A hybrid model featuring reprocessing in both the warm absorber/emitter and reflector may be the most realistic scenario. In this case, the preferred fit models the soft X-ray regime (and spectral variability) with the warm absorber/emitter, and the iron $\mathrm{K} \alpha$ line with a weakly ionized reflector $(\xi \lesssim 20)$. Full details will be presented elsewhere (Turner et al. 1996).

\section{References}

Turner, T. J., George, I. M., Kallman, T., Yaqoob, T., \& Życki, P. T. 1996, ApJ, 472 , in press. 